\title{
Software Design of Distribution Map Generation for Soil Parameters Based on VC++
}

\author{
Xiaofei $\mathrm{An}^{1,2, \mathrm{a}}$ Zhijun Meng ${ }^{1,2, \mathrm{~b}}$ Guangwei $\mathrm{Wu}^{1,2, \mathrm{c}} \quad$ Jianhua Guo ${ }^{1,2, \mathrm{~d}}$ \\ ${ }^{1}$ Beijing Research Center for Intelligent Agricultural Equipment, Beijing 100097, China; \\ 2 National Research Center of Intelligent Equipment for Agriculture, Beijing 100097, China. \\ aanxf@nercita.org.cn, ${ }^{b}$ mengzj@nercita.org.cn, ${ }^{c} w u g w @$ nercita.org.cn, \\ dguojh@nercita.org.cn
}

\begin{abstract}
Software system on distribution map generation of soil parameters was developed based on VC++. With the help of MapX control, soil spatial data could be edited, analyzed, generated distribution maps and so on. Soil parameter scatter graph was displayed and classified by equal count method, equal range method, natural break method and standard deviation method. In the software system soil parameter statistics data was also calculated and a new perimeter and area algorithm was developed. After comparison of different interpolation methods, Inverse Distance Weighted (IDW) method was selected to generate the soil parameter contour map and soil parameter iso-surface. At the same time the system gave the legend color and drawing method. These maps could also be saved as vector graphics. Finally a distribution map generation of soil parameters for soil moisture and total nitrogen was conducted by the soil samples from Xiangtang apple orchard in Beijing suburb, including scatter graph, contour map and iso-surface. The errors of perimeter and area were less than $3.72 \%$ and $3.01 \%$, respectively. The result shows that the system has perfect function and stable performance.
\end{abstract}

Keywords: Soil parameters, Distribution map, MapX control, Precision agriculture

\section{Introduction}

Soil is the basis of agricultural production, human has continued to research and analysis soil in the recent years. According to the theory of soil science, soil has two main functions: the first function is used to store water and supply crops; the second is to maintain the healthy growth of crop root. In order to describe the two functions, human use several parameters such as soil moisture, soil total nitrogen, soil fertility and many other parameters ${ }^{[1-4]}$. At the same time, domestic and foreign scholars have obtained a lot of results on the rapid detection of soil parameters ${ }^{[5-10]}$.

As a kind of new management ideas and modern agriculture, precision agriculture requires precise scientific fertilization and nutrient management ${ }^{[11-12]}$. In order to achieve the crop production in the process of scientific regulation measures of investment and management decisions, it must implement the precise fertilization 
decision-making. As a result it is particularly important to generating high precised soil parameter distribution map.

Currently, combinations of GIS tool software and visualization technology integrated have become the mainstream of the GIS application ${ }^{[13,14]}$. This article discussed the development of soil parameter distribution map system based on $\mathrm{VC}++$. With the help of MapX control ${ }^{[14-16]}$, it could display soil data, scatter plot and generate soil parameter distribution map.

\section{The structure of soil parameter distribution map system}

\subsection{Experimental Samples}

According to the system requirement, soil parameter distribution generation system mainly included the following modules: basic operation module, data import module, data statistics module and parameter map generation module. Figure 1 is the overall hierarchy diagram of soil parameter distribution generation system. In $\mathrm{VC}++6.0$ environment, the system has realized the import of soil parameter information, statistics and mapping, and other functions with MapX control.

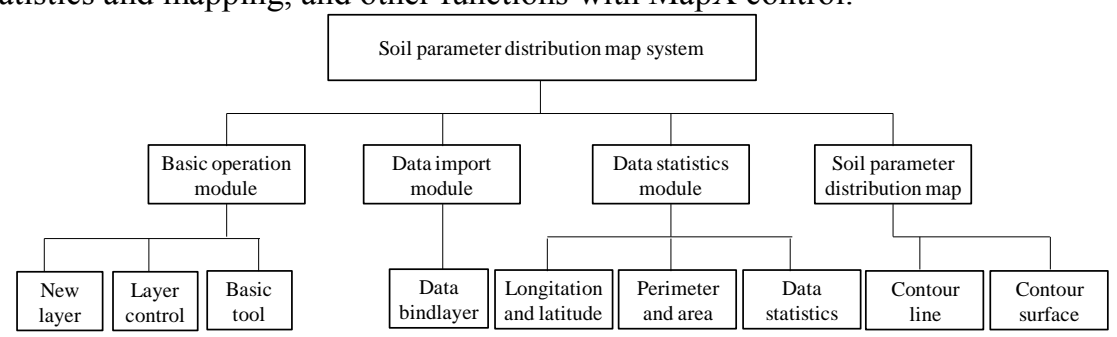

Fig.1 Overall structure of system

\section{Development of soil parameter distribution map system}

\subsection{Data binding}

Soil parameter information included soil moisture value, soil total nitrogen and soil GPS coordinates. Data binding is the process of soil parameter data from the data source into MapX [15]. In the MapX control, every map was corresponded to the multiple layers (the layers), and each layer (layer) have datasets, which contains a dataset object. Datasets with some properties and methods were mainly used to add and remove the dataset object in the collection. 
Soil parameter data mainly included GPS location information of soil and soil parameters attribute values. Then the binding layer type was set to miBindLayerTypeXY, which could realize to WGS54 coordinates $\mathrm{X}$ and $\mathrm{Y}$ coordinates of points. Figure 2 shows the imported the scatter plot after binding the soil moisture data.

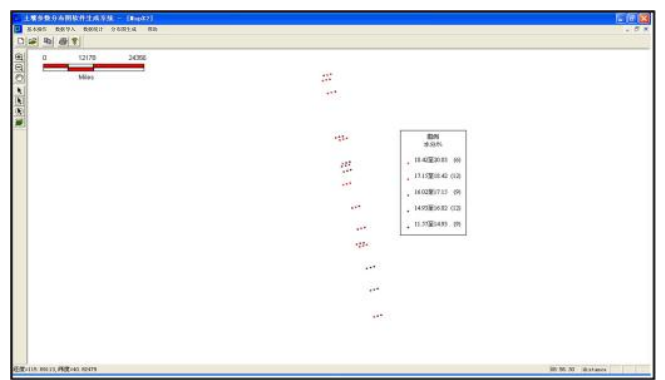

Fig.2 Data binding result

\subsection{Data classification and statistics}

After the soil data was binded to the soil layer, it could be classified by four methods, such as the counting method, the same range method, the nature devision method and the standard devision method. Figure 3 (a), (b), (c) and (d) shows the data classification results with four different methods.Not only the number could be statistics, but also the color of each rang of the legend could be edited.

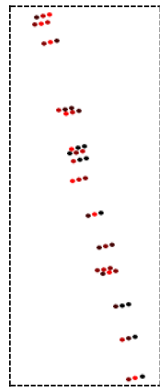

Moisture/o,

- 18.4-21.0 (9)

- 17.6-18.4 (9)

- $16.8-17.6(9)$

- 14.1-16.8 (11)

- 10.5-14.1 (10)

(a) The counting method

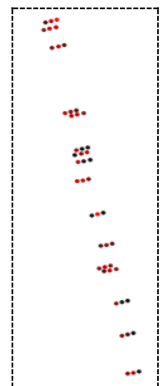

Moisture/\%

- 19.9-21.0 (2)

- $17.7-19.9(15)$

- $16.6-17.7$ (13)

- 15.4-16.6(7)

- $10.5-15.4$ (11)

(c) The nature devision method

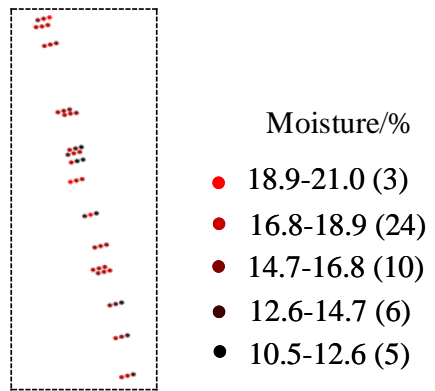

(b) The same range method

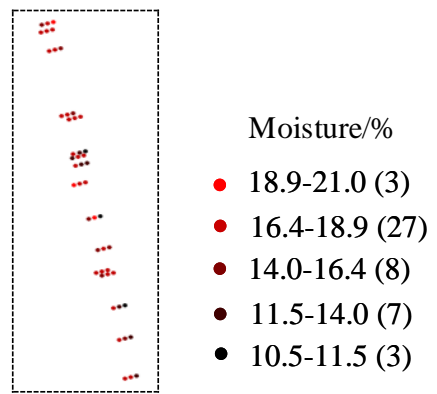

(d) The standard devision method 


\section{Fig.3 Data classification method}

It was said that soil and crop information always has a certain statistical regularity, although sometimes it does not get the distribution function of random variables. In this system the soil parameters could be calculate by maximum, minimum, the statistics of the mean and standard deviation. The mean value and mean square error of the calculation formula were shown in (1) and (2).

$$
\begin{aligned}
& \bar{v}=\frac{1}{n} \times \sum_{k=0}^{n-1} v_{k} \\
& \sigma=\sqrt{\frac{1}{n} \times \sum_{k=0}^{n-1}\left(\mathrm{v}_{k}-\bar{v}\right)^{2}}
\end{aligned}
$$

Where: $\sigma_{\text {was }}$ the data standard deviation value, $\bar{v}$ was the average of the data, $\mathrm{n}$ was the number of data points, $\mathrm{k}$ was the data point number, $v_{k}$ was the first $\mathrm{k}$ data of soil parameters.

\subsection{Perimeter and area calculation}

This system designed an algorithm to calculate perimeter and area with the direct use of GPS handheld device data. And projection method was directly to gaussian zoning projection. The center was east longitude $116^{\circ}$, projection of $40^{\circ}$.

MapX control provided map.distance method. This method could be used to calculate the linear distance between two points. Along the land border manually choose some key points, and then map.distance method was used repeatedly in sequence. Finally the perimeter was obtained accumulation. The system adopts the offset compensation method to calculate polygon area. Figure 4 was area calculation flow chart. 


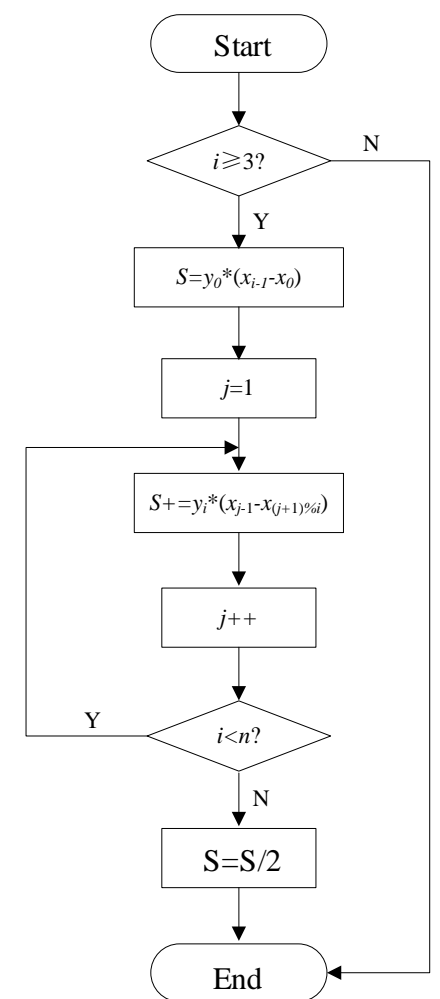

Fig.4 Area calculation flow chart

\subsection{Soil parameter distribution map generation}

With the steps of data binding, scatter plot, interpolation, contour map, soil parameter distribution map could be generated. By comparing the different types of spatial data interpolation methods, inverse distance weighting spatial data interpolation algorithm was finally chosen. After the original data interpolation was completed, the contour map was generated by the contour generating function provided by Contour.OCX control. Both the corresponding smoothness and Contour interval could be set. And finally the map could be saved format of SHP. Figure 5 was the distribution of soil parameters generated dialog.

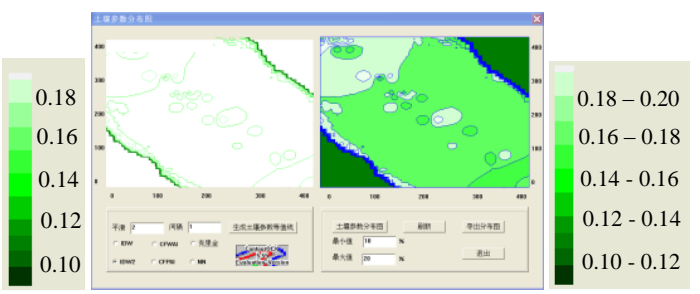

Fig.5 Soil parameter distribution map 


\section{Application of soil parameter distribution map system}

\subsection{Efficiency analysis}

To test the soil parameter distribution generation system efficiency, different sets of import test data was used. Choose the five sets of data, each group data included the number, soil parameter value latitude and longitude location information. Figure 6 is data import speed test results.

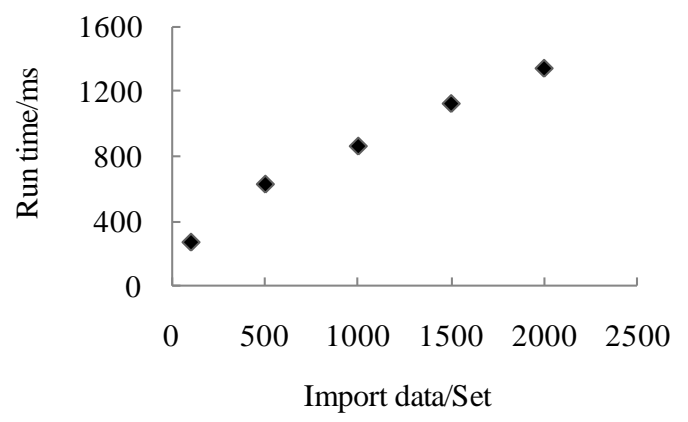

Fig.6 Data import speed result

It could be seen from the figure 6 , when import external data, this system increased with the amount of data. The consumption of time required to import the data were linearly increasing trend. When the data points was 2000 sets (the amount of data with 8000 ), the system took $1344 \mathrm{~ms}$. From the perspective of the function of angle and speed of system, functions could be fully realized, moderate speed in data quantity could meet the needs of practical application.

\subsection{Perimeter and area validation}

In order to test this system the perimeter area of the accuracy of the algorithm, experiments were carried out in Beijing changping apple orchards with GPS handheld instrument G738CM and the system. After sample the apple orchard with the device, the perimeter and area were calculated by the system. Table 1 and table 2 were the perimeter and area calculation error, respectively.

Tab.1 Perimeter calculation error

\begin{tabular}{ccccc}
\hline Number & Key point & $\begin{array}{c}\text { System } \\
\text { perimeter } / \mathrm{m}\end{array}$ & $\begin{array}{c}\text { System perimeter } \\
\text { with GPS device/m }\end{array}$ & error/\% \\
\hline 1 & 6 & 247.00 & 238.14 & 3.72 \\
2 & 8 & 246.81 & 238.00 & 3.70 \\
3 & 12 & 242.90 & 239.47 & 1.43 \\
\hline
\end{tabular}




\begin{tabular}{ccccc}
\hline 4 & 20 & 245.93 & 241.06 & 2.02 \\
5 & 28 & 245.58 & 242.14 & 1.42 \\
\hline Number & Key point & Tab.2 area calculation error & \\
\hline 1 & 6 & 2160.62 & $\begin{array}{c}\text { System area with } \\
\text { GPS device } / \mathrm{m}^{2}\end{array}$ & error/\% \\
\hline 2 & 8 & 2079.50 & 2097.40 & 3.01 \\
3 & 12 & 2110.55 & 2035.34 & 2.17 \\
4 & 20 & 2231.84 & 2087.28 & 1.11 \\
5 & 28 & 2248.96 & 2180.40 & 2.36 \\
\hline
\end{tabular}

It could be seen from table 1 and table 2, with the increase of the number of sampling points from 6 to 28 , apple orchards perimeter error reduced from $3.72 \%$ to $1.42 \%$, the average was 2.46\%; Area error reduced from $3.01 \%$ to $2.04 \%$, an average of $2.14 \%$. Key points directly affect the precision of the result. The more the key point was, the higher precision was. The error of the system was less than $3.72 \%$ and $3.01 \%$, respectively. It could satisfy practice.

\subsection{Soil parameter distribution map validation}

In order to verify the system operation, soil moisture and total nitrogen distribution maps of changing apple orchard were generated respectively, including the scatter plot, contour map and level surface. Inverse distance weighting spatial data interpolation method was used on the original data interpolation. The results showed that the system had stable performance. Figure 7 (a), (b), (c) and figure 8 (a), (b), (c) were the distributions of soil moisture and soil total nitrogen.

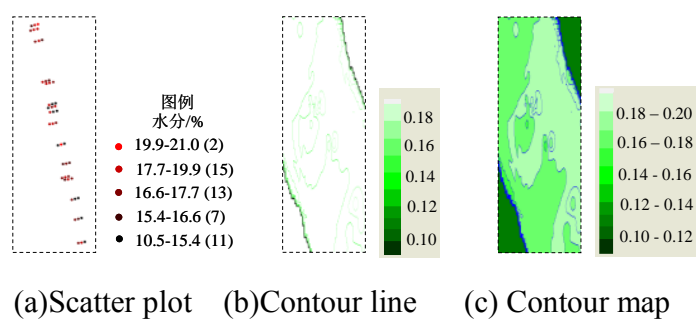

Fig.7 Soil moisture distribution map

\section{Conclusions}

Soil parameter distribution map system was developed based on $\mathrm{VC}++$. The system could realized the scatter plot, contour map, contour surface generation and vector graph save function. Through the analysis of the system efficiency and perimeter area 
computation verification test it showed that when the data points was 2000 , the timeconsuming needs $1344 \mathrm{~ms}$, calculation error within $3.72 \%$ and $3.01 \%$ respectively; The results showed that the system was stable and reliable enough. It provided a new means to guide the variable rate fertilization.

\section{Acknowledgment}

This paper was supported by the 863 programmar (2012AA101901) and 948 programmar(2014-G32).

\section{References}

1. Li Minzan. Spectral analysis and application[M]. Beijing, China: Science Press.2006.

2. Zheng Lihua. Real-time Sensing of Soil Parameters Based on Spectroscopy. [D], China Agricultural University, Beijing.2007.

3. Guo Jianhua, Zhao Chunjiang, Wang Xiu, et al. Research advancement and status on crop nitrogen nutrition diagnosis [J]. Soil and Fertilizer Sciences in China, 2008, (4):10 14.

4. Zhang Wangshou, Li Xiaoxiu, Huang Wenjiang, et al. Comprehensive assessment methodology of soil quality under different land use conditions[J]. Transactions of the CSAE, 2010, 26(12): 311 318.

5. Luo Xiwen, Zang Yin, Zhou Zhiyan. Research progress in farming information acquisition technique for precision agr iculture[J] . Transactions of the CSAE, 2006, 22(1):167 173.

6. He Dongjian, Yang Chenghai, Yang Qin, et al. Research progress of real-time measurement of soil attributes for precision agriculture[J]. Transactions of the CSAE, 2012, 28(7): 78 85.

7. Mouazen A M, Kuang B, De Baerdemaeker J, et al. On-line measurement of some selected soil properties using a VIS-NIR sensor[J]. Soil \& Tillage Research,2007,93(1):13 27.

8. Christy C D. Real-time measurement of soil attributes using on-the-go near infrared reflectance spectroscopy[J]. Computers and Electronics in Agriculture,2008,61:10 19.

9. Adamchuk V I, Hummel J W, Morgan M T, et al. On-the-go soil sensor for precision agriculture [J]. Computers and Electronics in Agriculture, 2004,(44):71 91.

10. Li Minzan, Pan Luan, Zheng Lihua, et al. Development of a portable SOM detector based on NIR diffuse reflection[J]. Spectroscopy and Spectral Analysis, 2010,30(4):1146 1150.

11. Wang Maohua. "Precision agriculture" with the practice of agricultural science and technology innovation [J]. China soft science, 1999, (4):21 25.

12. Wu Caicong. Research on the variable rate fertilization and economic analysis[D].Jilin University.2003.

13. Jin Hailiang, Liu Huijie, Miao Baoliang. Secondary development of GIS application software functions based on MapX controls [J].Computer \& Digital Engineering, 2011, 39(1):61 63.

14. He Yong, Fang Hui, Feng Lei. Information processing system for precision agriculture based on GPS and GIS [J]. 2002,18(1):145 149.

15. Yin Xure,Zhang Wujun. MapX technology based on viscal $\mathrm{C}++[\mathrm{M}]$.Beijing: Metallurgical industry press. 2009.

16. Yan Huiwu, Wu Xiaofang, Zhu Guorui. The development of GIS Application software on the basis of MapX control in VC++ [J]. Journal of Kunming University of Science and Technology, 2001, 26(6):12 17. 